\title{
Attitudes toward deprescribing for hospital inpatients
}

\author{
Authors: Richard Gilpin, ${ }^{A}$ Olwen C McDade ${ }^{B}$ and Chris Edwards ${ }^{C}$
}

\begin{abstract}
Clinicians are responsible for both commencing and stopping medications. This study evaluates the attitudes of older acute medical inpatients about deprescribing. Overall, patients are positive toward stopping medications, want to be involved and do not feel a clinician is giving up on them if a medication is stopped. Patients on fewer medications counterintuitively feel a greater medication burden, are more interested in being involved in decision making and consider deprescribing appropriate to a greater degree than patients who are taking more medications. Conversely, they also reported greater concerns about stopping medications. We discuss these findings in the context of the positive and negative effects of deprescribing, in the context of patient engagement and shared decision making, and how clinicians can work with inpatients to reduce potentially inappropriate medications.
\end{abstract}

KEYWORDS: deprescribing, medications, adverse effects

DOI: $10.7861 /$ clinmed.2021-0445

\section{Introduction}

Engaging in reducing a patient's medication burden is a common aspect of modern clinical practice. Medications that were once deemed appropriate may become less effective or even harmful, potentially causing adverse drug reactions. ${ }^{1}$ Deprescribing, "the process of withdrawal of an inappropriate medication, supervised by a health care professional with the goal of managing polypharmacy and improving outcomes', is a relatively new concept in healthcare. ${ }^{2}$ This involves the patient being included in a holistic shared decision-making process with the clinician to ensure medication efficacy. ${ }^{3}$

Stopping potentially inappropriate medications (PIM) has been shown to result in cognitive improvement, fewer falls and a positive change in a patient's global health, with no evidence of increased mortality. ${ }^{2,4-6}$ A systematic review of 31 deprescribing trials in older patients demonstrated that antihypertensive agents, psychotropic medicines and benzodiazepines could be discontinued without harm in between $20 \%$ and $100 \%$ of

Authors: ${ }^{\text {A }}$ specialist registrar in geriatric medicine, Nevill Hall Hospital, Abergavenny, UK; ${ }^{B}$ Patient and Carer Network representative, Royal College of Physicians, Cardiff, UK; ${ }^{{ }^{C}}$ Clinical scientist, Aneurin Bevan University Health Board, Newport, UK patients. ${ }^{7}$ Notably, ceasing statins in the final year of life improves quality of life with no effect on mortality. ${ }^{8}$ At least half of older patients may be taking at least one PIM. ${ }^{9,10}$

Deprescribing interventions have potential downsides; adverse drug withdrawal reactions, return of the medical condition, and pharmacokinetic and pharmacodynamic changes. ${ }^{11}$ If a patient feels well on their current medications, have been convinced that they need all of their medications, have a perceived lack of knowledge or are fearful about stopping, they may not be willing to engage in discussions. ${ }^{12,13}$ Clinicians are, therefore, encouraged to discuss the benefits and harms and engage in shared decision making when undertaking deprescribing. ${ }^{14}$

There is limited high-quality evidence to support clinical outcomes when deciding to cease a patient's medications, but the evidence available indicates that deprescribing interventions are safe and generally effective at reducing PIMs. ${ }^{15}$ Guides exist to support patients and clinicians; for example, the STOPP/ START guidelines or Beers' criteria have made progress in aiding clinicians' deprescribing decision making. ${ }^{16,17}$ A gap in how to approach the physician-patient shared decision-making process still exists, particularly for inpatients. ${ }^{14}$ Furthermore, deciding whether a medication is a PIM depends on the priorities of the patient. Is it important to reduce the risk of heart disease and stroke as much as possible, even when the side effects of the medication limit daily functioning? Patient narrative and involvement in this process is vital to establish and provide parameters to measure successful deprescribing.

Previous studies show that, although patients say they are satisfied with their existing medication, the majority are willing to engage with deprescribing. ${ }^{18-20}$ It is, therefore, important to explore how to involve the patient to ensure that the intervention of deprescribing is acceptable and effective. ${ }^{18}$

This study explores patients' attitudes to deprescribing by examining their responses within the context of their medication by using a standardised validated questionnaire in an inpatient setting. The study seeks to answer if a patient's attitude to deprescribing is related to the number of medications that they are prescribed, a relationship not studied previously for hospital inpatients. A previous community study showed no relation between number of medications prescribed and attitudes regarding deprescribing. ${ }^{12}$ Hospital admission provides an opportunity to review current medications, with a multidisciplinary approach, and the ability to observe the effects if the admission is of a suitable duration. The number of medications prescribed is a readily available numerical indicator for the admitting clinician. It has been estimated that around one in 10 hospital admissions are related to adverse drug reactions from prescription medications, and this invites the occasion to introduce deprescribing. ${ }^{19,20}$ 
Previous inpatient studies show patients agree with the concept of deprescribing, yet display significant variations to the extent in which they want to be involved. ${ }^{21,22}$

\section{Methods \\ Participants}

The survey was completed on the acute admission units across two local general hospitals in Aneurin Bevan University Health Board over a 4-month period. The team liaised with the nurse in charge to identify appropriate participants. Inclusion criteria were aged 65 years and over, mental capacity to participate, prescribed more than one medication and medically stable in order to participate (for example, not in pain or distress). Researchers did not identify their professional background and wore no clinical paraphernalia, with an aim to reduce potential bias.

\section{Design}

The standardised validated revised Patients' Attitudes Towards Deprescribing (rPATD) self-reporting questionnaire was used, which contained 22 statements with responses on a Likert scale, ranging from strongly agree to strongly disagree. ${ }^{23}$ Medications were recorded by class according to the British National Formulary. Participants were split into high and low medication users based on the mean number of medications, and evaluation was based on the proportion of 'agree' or 'strongly agree' responses to 'disagree' or 'strongly disagree' responses in each domain using a difference in proportions test. A $p<0.05$ was considered significant. Permission for this service evaluation was granted from Aneurin Bevan University Health Board's research and development team. Written consent was obtained.

\section{Results}

One-hundred and fifty-four participants were approached and 150 recruited, with four declining to undertake the questionnaire. Ninety-six $(64 \%)$ were women, with a mean age of 81 years. The median number of medications taken was nine; Table 1 lists the top 10 medications taken by class. Almost half ( $48.6 \%$ ) of participants were prescribed over 10 medications and six (4\%) were prescribed over 20.

Table 1. Proportion of patients taking at least one prescribed medication by class

$\begin{array}{ll}\text { Non-opiate analgesics } & 55 \% \\ \text { Opioids } & 44 \% \\ \text { Statins } & 43 \% \\ \text { Loop diuretics } & 37 \% \\ \text { Proton pump inhibitors } & 37 \% \\ \text { Vitamin D / calcium } & 35 \% \\ \text { Angiotensin converting enzyme inhibitors / angiotensin } & 33 \% \\ \text { receptor blocker } & \\ \text { Beta blockers } & 31 \% \\ \text { Osmotic laxatives } & 26 \% \\ \text { Antiplatelet } & 25 \%\end{array}$
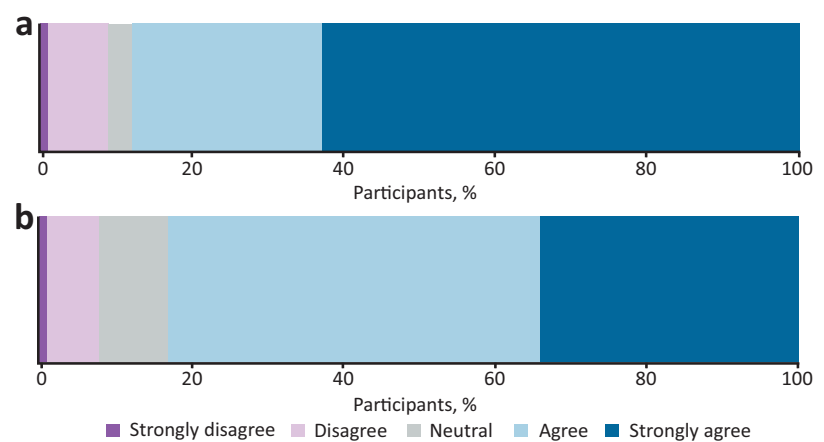

Fig 1. Responses to global statements. a) If my doctor said it was possible, I would be willing to stop one or more of my regular medicines. b) Overall, I am satisfied with my current medicines.

One-hundred and thirty-two (88\%) participants agreed or strongly agreed with global item 'If my doctor said it was possible, I would be willing to stop one or more of my regular medicines' and $126(84 \%)$ agreed or strongly agreed with the global item 'Overall, I am satisfied with my current medicines'. Fig 1 illustrates overall responses to the global items.

The participant groups were split into 'high' medication number and 'low' medication number using the mean number of medications of 10 as the threshold. There was no difference in gender or age between the groups. The mean number of medications for the 'low' group was 6.5 (range 2-9), and the mean for the 'high' group was 13.9 (range 10-25). Table 2 shows the responses to the rPATD based on medication use. Participants in the 'low' group reported a higher medication burden, considered deprescribing more appropriate and had a greater desire to be involved in decisions about deprescribing when compared with those in the 'high' group. Conversely, those in the 'low' group had greater concerns about stopping medications when compared with those in the 'high' group.

\section{Discussion}

This study set out to discover what the overall attitude to medications and deprescribing was for our older patients, and whether these views differed based on their medication burden. Participants arrived with a significant medication burden reflecting the patient group that is served in acute internal medicine.

Despite participants showing overall satisfaction with their medications, they appeared willing to engage in deprescribing. Participants showed a strong desire to be involved with decision making about prescribing and were less concerned about stopping. However, a greater proportion felt that their medications were not a burden and participants were equivocal about the appropriateness of deprescribing. Clinicians may be concerned that stopping a medication shows the patient that they are giving up on them; this was unfounded according to the results of this study. These responses to the global questions are comparable with previous studies using the rPATD in other environments, supporting the understanding that patients are willing to consider deprescribing medications. ${ }^{12,20,23-25}$

This is the first inpatient survey of deprescribing that has compared responses based on number of medications taken. What is notable is that those in the low medication use group still 
Table 2. Responses to the revised Patients' Attitudes Towards Deprescribing questionnaire by medication use

\begin{tabular}{|c|c|c|c|c|}
\hline & Disagree or strongly disagree & Agree or strongly agree & Mean score $^{a}(S D)$ & p value ${ }^{b}$ \\
\hline \multicolumn{5}{|l|}{ Burden } \\
\hline High & $40.3 \%$ & $52.9 \%$ & $16.9(4.0)$ & \\
\hline Low & $29.6 \%$ & $65.7 \%$ & $15.2(3.7)$ & 0.006 \\
\hline \multicolumn{5}{|c|}{ Appropriateness } \\
\hline High & $49.0 \%$ & $35.9 \%$ & $15.0(4.0)$ & \\
\hline Low & $39.2 \%$ & $47.0 \%$ & $13.5(4.4)$ & 0.032 \\
\hline \multicolumn{5}{|c|}{ Concerns about stopping } \\
\hline High & $31.5 \%$ & $60.6 \%$ & $18.2(3.4)$ & \\
\hline Low & $22.3 \%$ & $69.6 \%$ & $16.8(3.7)$ & 0.015 \\
\hline \multicolumn{5}{|c|}{ Involvement } \\
\hline High & $77.0 \%$ & $16.4 \%$ & $11.9(3.8)$ & \\
\hline Low & $66.0 \%$ & $26.8 \%$ & $10.0(3.3)$ & 0.001 \\
\hline
\end{tabular}

took a mean of over six medications. This means that the majority of this group's prescriptions were defined as polypharmacy (usually described as being on more than four medications). ${ }^{26}$ This group had more interest in being involved in decisions about their medications and were more likely to consider deprescribing appropriate. There are several explanations for this. It may be possible that the low medication group represents patients who are more involved, empowered and informed in their healthcare, ready to question and keen to limit their medication use, if possible. Another explanation might be that this group limit their interactions with healthcare due to better health literacy and so feel more empowered generally, plus the limitation of interactions leads to fewer prescribed medications. There is also likely to be multiple factors regarding a patient's medical background that have not been recorded by this survey.

With still restricted ability for patients to access specific deprescribing services or advice, the group taking a greater number of medications may represent patients who have not had the chance or stimulus to access advice rather than those who are unwilling to ever consider reducing their medication burden. This may explain the responses to the 'concerns about stopping' questions where, contrary to other aspects, those on a greater number of medications had fewer concerns about stopping medications. It is, therefore, unlikely that the results reflect a fear of 'upsetting the balance' for a highly medicated group with a potentially greater disease burden.

The findings of this study contrast with findings for patients in primary care; use of the rPATD in community-dwelling patients in Switzerland revealed no association in willingness to deprescribe and number of medications taken. ${ }^{12}$ The participants in this study were reasonably similar demographically, so it may indicate a feature of the environment that has influenced the difference of responses. Although, the authors note that they recruited only those with polypharmacy and they could not account for the attitudes of patients on fewer medications, as noted earlier, only a minority of participants in our study would not have fallen in the polypharmacy category.

There are several limitations in this study. The presence of a researcher may have biased participants' responses to the questions. Unlike other studies, we did not include the views of carers in this study as we wished to focus on patients with the capacity to make these decisions. It is likely that the advanced or proxy wishes of people without capacity may differ from the results of this study.

No consideration was made for whether the participant was taking a PIM, their clinical frailty score, or number and type of comorbidities. Whether a patient is on a PIM or not has been shown not to influence attitudes toward deprescribing, and it was beyond the scope of this study. ${ }^{9}$ A patient's comorbidities are very likely to affect their responses; a patient with a polysymptomatic chronic condition (such as Parkinson's disease) is very likely to have a different attitude toward their medications, but this study would not have been powered to recognise these idiosyncrasies. Further work evaluating interventions in specific conditions would need to take this understanding into consideration.

Research to date has noted that there exists no clear methodology to engaging in deprescribing, and that the process is complex. ${ }^{28}$

In addition to ceasing or reducing medications, deprescribing also encompasses using non-pharmacological approaches to care. ${ }^{29}$ This study did not explore how patients would be included in the approach to their care, or the behaviour changes associated with clinicians incorporating this into clinical practice as part of personalised care. Given the findings of this study, it is very likely that these approaches may need to be tailored based on several factors that may include medication number.

This study indicates that if you were undertaking work to practice safe deprescribing, then those patients taking under 10 medications are likely to be more engaged. The possibility that deprescribing may be more effective in patients taking fewer medications is contrary to previously published advice, and the authors feel that to draw this conclusion would be incorrect. ${ }^{14}$ The medications that a patient is prescribed will relate to where they are on a journey of comorbidity burden, clinical frailty and understanding of their future health risks and symptom control. Patients on a greater number of medications are likely to be at a later stage of this journey. Their circumstances are likely to have changed since a PIM was prescribed. We would, therefore, 
conclude that it is likely that a patient on a greater number of medications may require more engagement and education if a clinician wishes to consider deprescribing PIMs, and tailor their approach accordingly.

The main implication for future research is to underline the need to include patients in deprescribing discussions irrespective of number of medications, adding further evidence that hospital inpatients are generally willing to be included in deprescribing discussions. Hospital interventions focusing on deprescribing are, by their nature, time-consuming and intensive. ${ }^{6,15}$ This study suggests that different approaches may need to be undertaken depending on the medication burden of the individual, given the differences in responses discussed. Future research may determine the correct approach toward shared decision making based on this finding.

\section{Conclusion}

A cohort of older adults were largely supportive of their physician introducing the concept of deprescribing. They reported general satisfaction with their current medications but were willing to stop medications if this was possible. Those taking fewer than 10 medications considered deprescribing more appropriate and wanted to be involved in decisions but had greater concerns about stopping a medication compared with those taking 10 or more. We hope that this will lead to a better understanding of how to undertake shared decision making with patients to reduce medication burden and improve quality of life.

\section{Summary}

\section{What is known?}

Medications that were once deemed appropriate may become less effective or even harmful in older populations. Previous studies have shown that patients are generally in favour of appropriate deprescribing. No relationship between number of medications and deprescribing attitudes has been demonstrated in patients surveyed in the community.

\section{What is the question?}

What are the attitudes of older adult patients admitted to an acute hospital toward deprescribing, and is there a relationship between these attitudes and the number of medications being taken?

\section{What was found?}

The cohort of older adults expressed satisfaction with their current medications but were largely supportive of deprescribing. Patients on fewer medications counterintuitively felt a greater medication burden, are more interested in being involved in decision making and consider deprescribing appropriate to a greater degree than patients taking more medications. Conversely, they reported greater concerns about stopping medications.

\section{What is the implication for practice now?}

Patients admitted to hospital require their current medications to be transcribed, and this may be an opportunity for clinicians to consider whether these medications are appropriate. Overall, patients are willing to consider deprescribing potentially inappropriate medications. The number of medications a patient takes is likely to influence their engagement with deprescribing, and the clinician should be aware of this when engaging in shared decision making.

\section{References}

1 Lavan AH, Gallagher P. Predicting risk of adverse drug reactions in older adults. Therapeutic Advances in Drug Safety 2016;7:11-22.

2 Reeve E, Gnjidic D, Long J, Hilmer S. A systematic review of the emerging definition of 'deprescribing' with network analysis: implications for future research and clinical practice. British Journal of Clinical Pharmacology 2015;80:1254-68.

3 Sloane PD, Zimmerman S. Deprescribing in geriatric medicine: challenges and opportunities. Journal of the American Medical Directors Association 2018;19:919-22.

4 Krishnaswami A, Steinman MA, Goyal P et al. Deprescribing in older adults with cardiovascular disease. Journal of the American College of Cardiology 2019;73:2584-95.

5 Potter K, Flicker L, Page A, Etherton-Beer C. Deprescribing in frail older people: a randomised controlled trial. PloS One 2016;11:e0149984.

6 Edey R, Edwards N, Von Sychowski ] et al. Impact of deprescribing rounds on discharge prescriptions: an interventional trial. International Journal of Clinical Pharmacy 2019;41:159-66.

7 Iyer S, Naganathan V, McLachlan AJ, Le Conteur DG. Medication withdrawal trials in people aged 65 years and older. Drugs \& Aging 2008;25:1021-31.

8 Kutner JS, Blatchford PJ, Taylor DH et al. Safety and benefit of discontinuing statin therapy in the setting of advanced, lifelimiting illness: a randomized clinical trial. JAMA Internal Medicine 2015;175:691-700.

9 Achterhof $A B$, Rozsnyai Z, Reeve E et al. Potentially inappropriate medication and attitudes of older adults towards deprescribing. PloS One 2020;15:e0240463.

10 Gallagher P, Lang PO, Cherubini A et al. Prevalence of potentially inappropriate prescribing in an acutely ill population of older patients admitted to six European hospitals. European Journal of Clinical Pharmacology 2011:67:1175-88.

11 Reeve E, Shakib S, Hendrix I, Roberts MS, Wiese MD. The benefits and harms of deprescribing. Medical Journal of Australia 2014:201:386-9.

12 Rozsnyai Z, Jungo KT, Reeve $\mathrm{E}$ et al. What do older adults with multimorbidity and polypharmacy think about deprescribing? The LESS study-a primary care-based survey. BMC Geriatrics 2020;20:1-11.

13 Belcher VN, Fried TR, Agostini JV, Tinetti ME. Views of older adults on patient participation in medication-related decision making. Journal of General Internal Medicine 2006;21:298-303.

14 Jansen J, Naganathan V, Carter SM et al. Too much medicine in older people? Deprescribing through shared decision making. BMJ 2016;353:i2893.

15 Thillainadesan ], Gnjidic D, Green S, Hilmer SN. Impact of deprescribing interventions in older hospitalised patients on prescribing and clinical outcomes: a systematic review of randomised trials. Drugs \& Aging 2018;35:303-19.

16 O'Mahony D, O'Sullivan D, Byrne $S$ et al. STOPP/START criteria for potentially inappropriate prescribing in older people: version 2. Age and Ageing 2015;44:213-8.

17 Fick DM, Cooper JW, Wade WE et al. Updating the Beers criteria for potentially inappropriate medication use in older adults: results of a US consensus panel of experts. Archives of Internal Medicine 2003:163:2716-24.

18 Orom H, Underwood IIIW, Cheng Z et al. Relationships as medicine: quality of the physician-patient relationship determines physician influence on treatment recommendation adherence. Health Services Research 2018;53:580-96.

19 Oscanoa TJ, Lizaraso F, Carvajal A. Hospital admissions due to adverse drug reactions in the elderly. A meta-analysis. European Journal of Clinical Pharmacology 2017;73:759-70.

20 Reeve E, Wolff JL, Skehan M et al. Assessment of attitudes toward deprescribing in older Medicare beneficiaries in the United States. JAMA Internal Medicine 2018;178:1673-80. 
21 Scott IA, Hilmer SN, Reeve E et al. Reducing inappropriate polypharmacy: the process of deprescribing. JAMA Internal Medicine 2015;175:827-34.

22 Komagamine J, Sugawara K, Hagane K. Characteristics of elderly patients with polypharmacy who refuse to participate in an inhospital deprescribing intervention: a retrospective cross-sectional study. BMC Geriatrics 2018;18:1-6.

23 Reeve E, Low LF, Shakib S, Hilmer SN. Development and validation of the revised patients' attitudes towards deprescribing (rPATD) questionnaire: versions for older adults and caregivers. Drugs \& Aging 2016;33:913-28.

24 Reeve E, Low LF, Hilmer SN. Attitudes of older adults and caregivers in Australia toward deprescribing. Journal of the American Geriatrics Society 2019:67:1204-10.

25 Scott S, Clark A, Farrow C et al. Attitudinal predictors of older peoples' and caregivers' desire to deprescribe in hospital. BMC Geriatrics 2019;19:108.

26 Masnoon N, Shakib S, Kalisch-Ellett L, Caughey GE. What is polypharmacy? A systematic review of definitions. BMC Geriatrics 2017:17:230
27 Ammerman CA, Simpkins BA, Warman N, Downs TN. Potentially inappropriate medications in older adults: Deprescribing with a clinical pharmacist. Journal of the American Geriatrics Society 2019:67:115-8.

28 Hansen CR, O'Mahony D, Kearney PM et al. Identification of behaviour change techniques in deprescribing interventions: a systematic review and meta-analysis. British Journal of Clinical Pharmacology 2018;84:2716-28.

29 Farrell B, Conklin J, Dolovich L et al. Deprescribing guidelines: An international symposium on development, implementation, research and health professional education. Research in Social and Administrative Pharmacy 2019;15:780-9.

Address for correspondence: Dr Richard Gilpin, Nevill Hall Hospital, Aneurin Bevan University Health Board, Brecon Road, Abergavenny NP7 7EG, UK.

Email: richardgilpin@doctors.org.uk 